\title{
MODELING DUST FRAGMENTATION IN COMETS
}

\author{
I. KONNO and W.F. HUEBNER \\ Southwest Research Institute \\ P.O. Drawer 28510 \\ San Antonio, TX 78228-0510 \\ USA
}

\begin{abstract}
We developed a 1-D hydrodynamic model of dusty gas flow with dust fragmentation in a cometary atmosphere and performed calculations for a dust-size distribution with radii $a=10^{-4}$ $10 \mathrm{~cm}$ and densities variable with dust size. A comparison was made with Giotto observations of dust jet intensities within $100 \mathrm{~km}$ of the nucleus of Comet Halley. We found that dust fragmentation cannot be solely responsible for the flattening of the dust intensity near the nucleus with respect to the $1 / R$ law. We conclude that a combination of geometric effects and grain fragmentation may explain the observed intensity profiles.
\end{abstract}

\section{Introduction}

Dust intensity profiles obtained by the Halley Multicolour Camera (HMC) show a sharp deviation from the expected $1 / R$ dependence ( $R$ is the cometocentric distance). Thomas et al. (1988), Huebner et al. (1988), and Reitsema et al. (1989) have successfully used geometric effects to explain the deviation. However, Szegö et al. (1988) found the deviation seen in profiles using Vega images to be too large to be explained by source geometry effects alone and suggested dust particle fragmentation as an alternative. From Giotto's PIA and DIDSY data, McDonnell et al. (1987) found some evidence for possible fragmentation or evaporation of grains. Vaisberg et al. (1987) obtained several pieces of evidence for an evolution (including splitting) of the grains during their motion in the cometary coma.

\section{Modeling}

We solve the hydrodynamic equations for a dusty gas flow in a comet coma. The fragmentation of dust particles into the next smaller size is incorporated in the model by adding source and sink terms in the continuity equations for the dust. The lifetime $\tau_{i}$ of dust particle with radius $a_{i}\left(a_{1}\right.$ is the minimum size) is given by

$$
\tau_{i}=\tau_{\max }\left[\log \left(a_{i} / a_{\max }\right)\right]^{\alpha},
$$

where $\tau_{\max }$ is the lifetime of the particle of the maximum size $a_{\max }$. The lifetime of the smallest particles is assumed infinite. The size distribution at the nucleus surface is given by

$$
\rho_{i}=\rho_{\max }\left(a_{i} / a_{\max }\right)^{\beta}
$$

where $\rho_{i}$ is the spatial mass density of the particles of radius $a_{i}$ and $\rho_{\max }$ is the density of the particles of the maximum radius $a_{\max }$ that can be entrained by the gas. The bulk density of dust particles is a function of dust size (Lamy et al., 1987):

A.C. Levasseur-Regourd and H. Hasegawa (eds.), Origin and Evolution of Interplanetary Dust, $221-224$. (c) 1991 Kluwer Academic Publishers, Printed in Japan. 


$$
\rho_{b u l k}=2.2-1.4 a /\left(a_{0}+a\right) \mathrm{g} \mathrm{cm}^{-3}, a_{0}=10^{-4} \mathrm{~cm} .
$$

We perform calculations with dust particles of radius $10^{-4} \mathrm{~cm}$ to $10 \mathrm{~cm}$ with a continuous distribution approximated by 26 discrete particle sizes seperated by a logarithmic scale, so that a particle always fragments into about four particles of the next smaller size. We define the mass density by $\rho$, velocity by $v$, pressure by $P$, ratio of specific heats by $\gamma$, number density by $n$, temperature by $T$. The subscripts $g$ and $d$ refer to gas and dust, respectively.

The hydrodynamic equations for the single-fluid, inviscid, perfect gas are as follows.

$$
\begin{gathered}
\frac{1}{R^{2}} \frac{d}{d R}\left(R^{2} \rho_{g} v_{g}\right)=\dot{\rho}_{g}, \\
\rho_{g} v_{g} \frac{d v_{g}}{d R}+\frac{d P_{g}}{d R}=-\sum_{i} F_{g d, i}, \\
\frac{1}{\gamma-1}\left(v_{g} \frac{d \rho_{g}}{d R}\right)+\frac{\gamma}{\gamma-1} \rho_{g} \frac{d v_{g}}{d R}=\dot{Q}_{p h o t o}-\sum_{i} \dot{Q}_{g d, i},
\end{gathered}
$$

where $F_{g d, i}$ is the momentum transfer rate from the gas to the dust of size $a_{i}$, given by

$$
F_{g d, i}=\frac{1}{2} C_{D, i} \pi a^{2} \rho_{g}\left(v_{g}-v_{d, i}\right)^{2} n_{d, i} .
$$

Here $C_{D, i}$ is the modified free molecular drag coefficient defined as follows (cf. Probstein, 1968):

$$
C_{D, i}=\frac{2 \sqrt{\pi}}{3} \sqrt{\frac{T_{d}}{T_{g}}}+\frac{2 s_{i}^{2}+1}{s_{i}^{2} \sqrt{\pi}} e^{-s_{i}^{2}}+\frac{4 s_{i}^{4}+4 s_{i}^{2}-1}{2 s_{i}^{3}} \operatorname{erf}\left(s_{i}\right), \quad \vec{s}_{i}=\frac{\vec{v}_{g}-\vec{v}_{d, i}}{\sqrt{2 k T_{g} / m_{g}}},
$$

where $k$ is the Boltzmann constant and $m_{g}$ is the molecular mass of the gas. $\dot{Q}_{\text {photo }}$ is the rate of heating of the gas due to the photodissociation of water and $\dot{Q}_{g d, i}$ is the energy tranfer rate from the gas to the dust. At distances $R<<10^{4} \mathrm{~km}, \dot{\rho}_{g}$ for $\mathrm{H}_{2} \mathrm{O}$ is negligible, so that we set $\dot{\rho}_{g}=0$ in the application in the next section.

For the dust, the following hydrodynamic equations are solved for each dust particle of size $a_{i}$.

$$
\begin{gathered}
\frac{1}{R^{2}} \frac{d}{d R}\left(R^{2} \rho_{d, i} v_{d, i}\right)=-\frac{\rho_{d, i}}{\tau_{i}}+\frac{\rho_{d, i+1}}{\tau_{i+1}} \\
\rho_{d, i} v_{d, i} \frac{d v_{d, i}}{d R}-\rho_{d, i} g_{c o m e t}(R)=F_{g d, i} \\
\rho_{d, i} v_{d, i} C_{D, i} \frac{d T_{d, i}}{d R}=\dot{Q}_{g d, i}+\dot{Q}_{r a d, i}
\end{gathered}
$$

where $g_{\text {comet }}(R)$ is the gravitational acceleration by the comet nucleus and $\dot{Q}_{r a d, i}$ is the rate of the radiation energy due to solar heating and IR cooling.

We assume for the nucleus of Comet Halley that the effective radius $R_{n}=4.0 \mathrm{~km}$, the density $\rho_{n}=0.5 \mathrm{~g} \mathrm{~cm}^{-3}$, and the dust-to-gas mass ratio $\chi=1.0$. The reason that the nucleus density is lower than the average dust density is that the material in the cometary nucleus may be more porous than dust particles and contains hollow space.

\section{Application: Dust Jets near the Nucleus of Comet Halley}

We apply the dust fragmentation model to the problems of the dust intensity profiles near the nucleus of Comet Halley. Because cometary dust is optically thin, the observed dust intensity, which is proportional to the dust column density, should change like $1 / R$. However, intensity 
profiles of jet-like features analyzed in the HMC images from the Giotto spacecraft (Thomas et al., 1988; Huebner et al., 1988) show strong flattening relative to a $1 / R$ profile near the nucleus.

Thomas and Keller (1990) have explained the dust intensity profile using a dust fragmentation calculation. They showed that on the average, fragmentation of a particle into 2.7 particles can explain the $30 \%$ increase of $I \cdot R$ (where $I$ is the scattered light intensity) beyond $9 \mathrm{~km}$ from the source.

The dust intensity profiles in Figure 1 obtained by Huebner et al. (1988) show a steep rise of $I \cdot R$ within $50-100 \mathrm{~km}$ from the source. They have shown that a wide range of dust profiles can be modeled using different source geometries and obtained qualitative agreement with HMC profiles without invoking other mechanisms. Although their model fit the data very well, it assumes a constant velocity for the dust particles. Dust particles are accelerated by gas-drag force from rest to the terminal velocity of several $100 \mathrm{~km} \mathrm{~s}^{-1}$ to $1.0 \mathrm{~km} \mathrm{~s}^{-1}$, depending on their size. Figure 2 shows an $I \cdot R$ profile obtained from a hydrodynamic calculation with the dust size distribution given by equation (10) with $\beta=1.0$ in the case of no fragmentation. The figure shows a rapid decrease of $I \cdot R$, which is just the opposite of the observed profiles. Therefore, models for the observed dust in tensity profile must account for the dust acceleration.

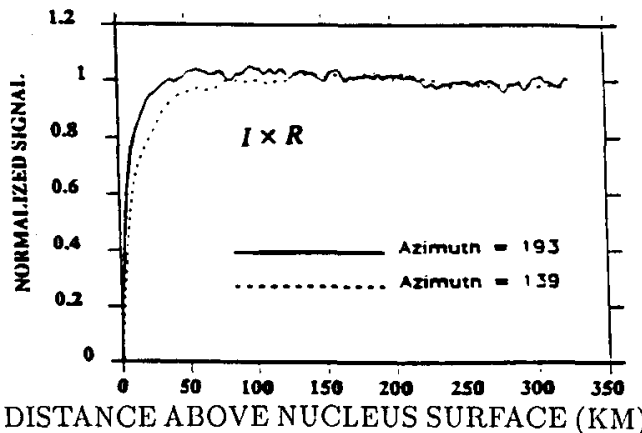

Fig. $1-I \cdot R$ profiles for dust obtained by the Giotto spacecraft. If the intensity $I \sim 1 / R$, then $I \cdot R$ should be constant. From Huebner et al. (1988).

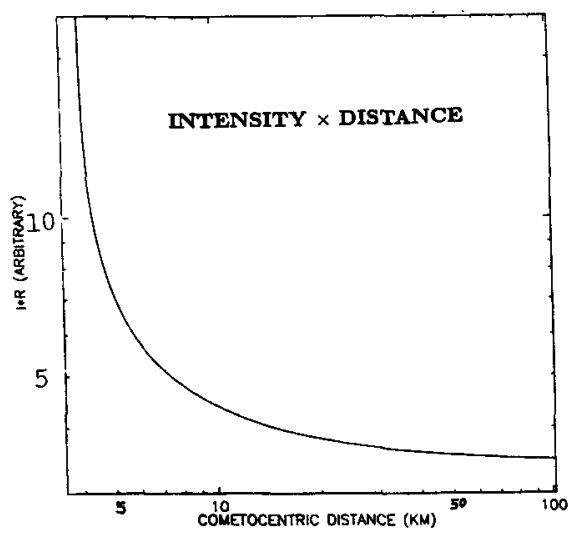

Fig. 2 - A calculated $I \cdot R$ profile for dust when there is no dust fragmentation. $I \cdot R$ is not constant because of acceleration.

We performed the hydrodynamic calculations to see if we can explain the observed dust profiles by dust fragmentation (Figure 3 ). The parameters for the model are $\alpha=0, \beta=1.0, \tau_{\max }=10$ sec. The model seems to fit the observation fairly well. The lifetime for the dust particles used for the fit, however, is much too short for particles to survive. Figure 4 shows the dust density for several sizes as a function of cometocentric distance. We see that all particles fragment into the smallest ones within $50 \mathrm{~km}$, which is contradiction to observations because larger particles have been detected at much larger distances.

The fragmentation calculation by Thomas and Keller (1990) gives the lower limit to fragmentation since their profile does not include the region closer than $\sim 9 \mathrm{~km}$ to the nucleus. We conclude that fragmentation alone cannot be responsible for the deviation of the dust intensity profile from $1 \mathrm{~km}$ to $\sim 50 \mathrm{~km}$ near the nucleus observed by the Giotto spacecraft. The combination of the geometric effect (Huebner et al., 1988) and dust fragmentation described here may explain the deviation. 


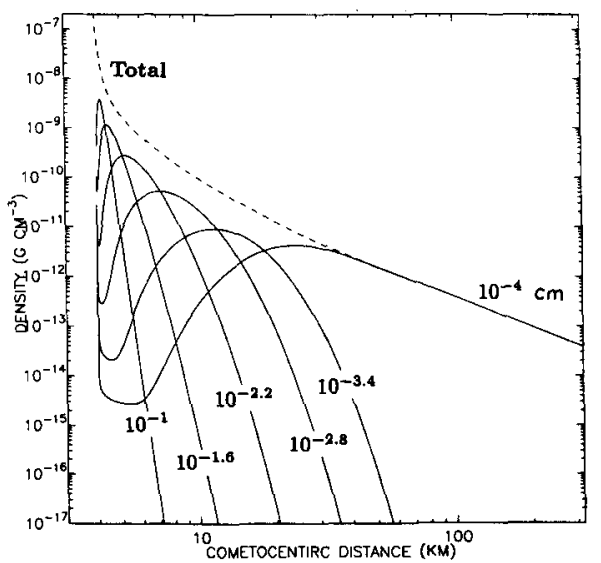

Fig. 3 - Model $I \cdot R$ profile for dust compared with the observation, the solid line in Figure 1.

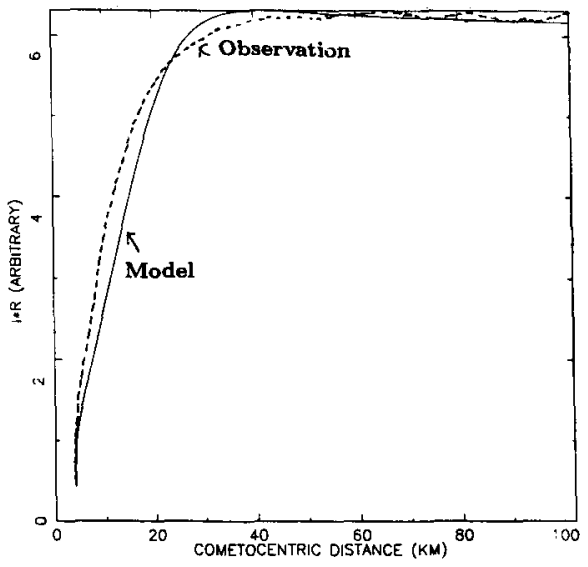

Fig. 4 - Dust space density obtained for the model in Figure 3.

\section{References}

Huebner, W.F., Boice, D.C., Reitsema, H.J., Delamere, W.A., and Whipple, F.L. (1988) 'A model for intensity profiles of dust jets near the nucleus of comet Halley', Icarus, 76, 78-88.

Lamy, P.L., Grün, E., and Perrin, J.M. (1987) 'Comet P/Halley: implications of the mass distribution function for the photometric properties of the dust coma', Astron. Astrophys. 187, 767-773.

McDonnell, J.A.M. et al. (1987) 'The dust distribution within the inner coma of comet P/Halley 1982i: encounter by Giotto's impact detectors', Astron. Astropys. 187, 719-741.

Probstein, R.F. (1968) 'The dusty gas dynamics of comet heads', Prablems of Hydrodynamics and Continuum Mechanics, Soc. Industr. Apply. Math., 568-583.

Reitsema, H.J., Delamere, W.A., Williams, A.R., Boice, D.C., Huebner, W.F., and Whipple, F.L. (1989) 'Dust distribution in the inner coma of comet Halley: comparison with models', Icarus, 81, 31-40.

Szegö, K., Tóth, I., Szatmáry, Z., Smith, B.A., Kondor, A., and Merényi (1988) 'Dust photometry in the near nucleus region of comet Halley", preprint, KFKI-1988-33/C

Vaisberg, O.L., Smirnov, V., Omelchenko, A., Gorn, L., and Iovlev, M. (1987) 'Spatial and mass distribution of low-mass dust particles $\left(\mathrm{m}<10^{-10} \mathrm{~g}\right)$ in comet P/Halley's coma', Astron. Astrophys. 187, 753-760.

Thomas, N. and Keller, H.U. (1987) 'Comet P/Halley's near nucleus jet activity', in Symposium on the diversity and similarity of comets, ESA SP-278, 337-342.

Thomas, N. and Keller, H.U. (1990) 'Interpretation of the inner coma observations of comet P/Halley multicolor camera', Annales Geophysicae, 8, (2), 147-166.

Thomas, N., Boice, D.C., Huebner, W.F., and Keller, H.U. (1988) 'Intensity profiles of dust near extended sources on comet Halley', Nature, 332, 51-52. 